Supporting Information for

\title{
Ammonium Salt-Accelerated Hydrazinolysis of Unactivated Amides: Mechanistic Investigation and Application to Microwave Flow Process
}

Megumi Noshita, ${ }^{\dagger}$ Yuhei Shimizu, ${ }^{\dagger}$ Hiroyuki Morimoto $, *,{ }^{\dagger}$ Shuji Akai,,${ }^{\star}$ Yoshitaka Hamashima, ${ }^{\S}$ Noriyuki Ohneda, ${ }^{\|}$Hiromichi Odajima, ${ }^{\perp}$ and Takashi Ohshima*,†

†Graduate School of Pharmaceutical Sciences, Kyushu University, Fukuoka, 8128582, Japan.

*Graduate School of Pharmaceutical Sciences, Osaka University, Suita, 567-0871, Japan.

${ }^{\S}$ School of Pharmaceutical Sciences, University of Shizuoka, Suruga-ku, Shizuoka, Shizuoka 422-8526, Japan.

"SAIDA FDS INC., 143-10, Isshiki, Yaizu, Shizuoka, 425-0054, Japan.

${ }^{\perp}$ Pacific Microwave Technologies Corp., Seattle, USA.

E-mail address: ohshima@phar.kyushu-u.ac.jp,hmorimot@phar.kyushu-u.ac.jp

\section{Table of Contents}

1. Detailed Information for Calculated Structures $\quad$ S2

$\begin{array}{lll}\text { 2. Spectral Data } & \text { S22 }\end{array}$ 


\section{Detailed Information for Calculated Structures}

\section{Starting Materials}

\section{AcNHMe}

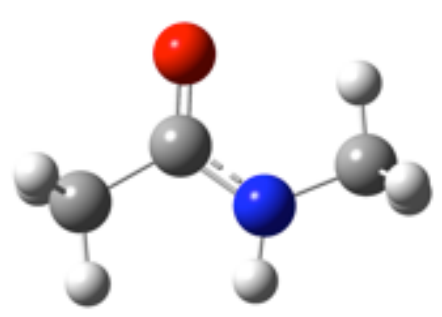

B3LYP/6-31G(d) free energy: -248.451404 (a.u.)

B3LYP/6-31G(d) thermal correction to Gibbs free energy: 0.072159 (a.u.)

Number of imaginary frequencies: 0

M06-2X/6-311+G(d,p)/SMD $\left(\mathrm{H}_{2} \mathrm{O}\right)$ single point energy: -248.4992988 (a.u.)

M06-2X/6-311+G(d,p)/SMD(EtOH) single point energy: -248.500761 (a.u.)

M06-2X/6-311+G(d,p)/SMD $\left(\mathrm{CF}_{3} \mathrm{CH}_{2} \mathrm{OH}\right)$ single point energy: -248.498819922 (a.u.)

$\begin{array}{lrrr}\mathrm{C} & -0.48337500 & 0.16817200 & -0.00001800 \\ \mathrm{C} & -1.81705400 & -0.56683900 & 0.00001800 \\ \mathrm{H} & -2.38817300 & -0.25913900 & 0.88130800 \\ \mathrm{H} & -2.38820500 & -0.25927200 & -0.88129700 \\ \mathrm{H} & -1.72520800 & -1.65795100 & 0.00009700 \\ \mathrm{O} & -0.42201100 & 1.39125900 & -0.00000400 \\ \mathrm{~N} & 0.62527500 & -0.63508400 & -0.00005000 \\ \mathrm{H} & 0.49912900 & -1.63617900 & 0.00001400 \\ \mathrm{C} & 1.97546000 & -0.10354700 & 0.00002400 \\ \mathrm{H} & 2.52967800 & -0.42155800 & -0.89142700 \\ \mathrm{H} & 1.89223600 & 0.98425100 & -0.00010700 \\ \mathrm{H} & 2.52951500 & -0.42135200 & 0.89165300\end{array}$

\section{$\mathrm{H}_{2} \mathrm{NNH}_{2}$}

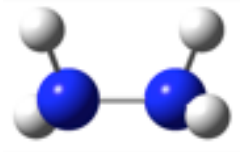

B3LYP/6-31G(d) free energy: -111.825575 (a.u.)

B3LYP/6-31G(d) thermal correction to Gibbs free energy: 0.030871 (a.u.)

Number of imaginary frequencies: 0

M06-2X/6-311+G(d,p)/SMD $\left(\mathrm{H}_{2} \mathrm{O}\right)$ single point energy: -111.8605073 (a.u.) 
M06-2X/6-311+G(d,p)/SMD(EtOH) single point energy: -111.8607171 (a.u.) M06-2X/6-311+G(d,p)/SMD( $\left.\mathrm{CF}_{3} \mathrm{CH}_{2} \mathrm{OH}\right)$ single point energy: -111.8606261 (a.u.)

$\begin{array}{lrrc}\mathrm{N} & -0.71114800 & -0.07742300 & 0.10307600 \\ \mathrm{H} & -1.05658700 & 0.84764300 & 0.36651300 \\ \mathrm{~N} & 0.71115000 & -0.07755300 & -0.10298300 \\ \mathrm{H} & 1.05659600 & 0.84718600 & -0.36754500 \\ \mathrm{H} & 1.13584100 & -0.30450700 & 0.79315200 \\ \mathrm{H} & -1.13586300 & -0.30549000 & -0.79276900\end{array}$

\section{$\mathrm{H}_{2} \mathrm{NNH}_{3}^{+}$}

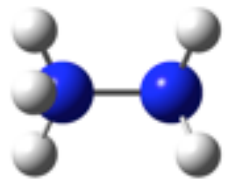

B3LYP/6-31G(d) free energy: -112.159172 (a.u.)

B3LYP/6-31G(d) thermal correction to Gibbs free energy: 0.045381 (a.u.) Number of imaginary frequencies: 0

M06-2X/6-311+G(d,p)/SMD $\left(\mathrm{H}_{2} \mathrm{O}\right)$ single point energy: -112.3161372 (a.u.) $\mathrm{M} 06-2 \mathrm{X} / 6-311+\mathrm{G}(\mathrm{d}, \mathrm{p}) / \mathrm{SMD}(\mathrm{EtOH})$ single point energy: -112.314724 (a.u.) M06-2X/6-311+G(d,p)/SMD $\left(\mathrm{CF}_{3} \mathrm{CH}_{2} \mathrm{OH}\right)$ single point energy: -112.3149183 (a.u.)

$\begin{array}{lrrc}\mathrm{N} & 0.78088200 & 0.00000100 & -0.12393900 \\ \mathrm{H} & 1.13780600 & 0.82898600 & 0.35326700 \\ \mathrm{~N} & -0.66268300 & -0.00000500 & 0.01867800 \\ \mathrm{H} & -1.04001700 & -0.00089400 & 0.98230200 \\ \mathrm{H} & -1.03142900 & 0.82453300 & -0.47518000 \\ \mathrm{H} & 1.13774800 & -0.82903800 & 0.35321200 \\ \mathrm{H} & -1.03150400 & -0.82355600 & -0.47677700\end{array}$

Structures for the Reaction Pathway in the Presence of Hydrazinium Salt I (AcNHMe with $\mathrm{H}_{2} \mathrm{NNH}_{2}$ and $\mathrm{H}_{2} \mathrm{NNH}_{3}{ }^{+}$) 


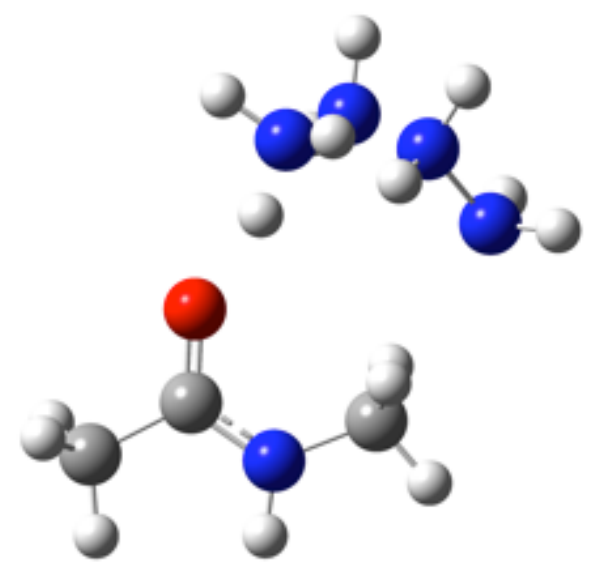

B3LYP/6-31G(d) free energy: -472.493568 (a.u.)

B3LYP/6-31G(d) thermal correction to Gibbs free energy: 0.18425 (a.u.)

Number of imaginary frequencies: 0

M06-2X/6-311+G(d,p)/SMD $\left(\mathrm{H}_{2} \mathrm{O}\right)$ single point energy: -472.699790582 (a.u.)

M06-2X/6-311+G(d,p)/SMD(EtOH) single point energy: -472.70272198 (a.u.)

M06-2X/6-311+G(d,p)/SMD $\left(\mathrm{CF}_{3} \mathrm{CH}_{2} \mathrm{OH}\right)$ single point energy: -472.701206354 (a.u.)

$\begin{array}{lrrr}\mathrm{C} & 2.21611900 & -0.09794900 & 0.19113500 \\ \mathrm{C} & 3.57020900 & -0.52471900 & 0.70874400 \\ \mathrm{H} & 3.47344000 & -0.81859200 & 1.75843000 \\ \mathrm{H} & 3.89980600 & -1.40767400 & 0.15260000 \\ \mathrm{H} & 4.33339000 & 0.25330400 & 0.62564600 \\ \mathrm{O} & 1.25707500 & -0.89607600 & 0.21590100 \\ \mathrm{H} & -0.34715300 & -1.05387500 & 0.20611900 \\ \mathrm{~N} & 2.10372800 & 1.14969100 & -0.29203900 \\ \mathrm{H} & 2.92501300 & 1.73789300 & -0.29149300 \\ \mathrm{C} & 0.87056800 & 1.68132200 & -0.86209100 \\ \mathrm{H} & 0.53757600 & 1.06063300 & -1.70067800 \\ \mathrm{H} & 0.06875300 & 1.72548600 & -0.11814800 \\ \mathrm{H} & 1.06574000 & 2.69007800 & -1.22859800 \\ \mathrm{H} & -2.88632700 & 0.71073200 & 1.98970300 \\ \mathrm{H} & -4.12063800 & 0.24925000 & 1.01141700 \\ \mathrm{~N} & -2.66796300 & 1.44318600 & 0.14794700 \\ \mathrm{~N} & -3.10788800 & 0.40163000 & 1.04381800 \\ \mathrm{H} & -3.32196000 & 2.22711100 & 0.15565000 \\ \mathrm{~N} & -1.31051600 & -1.49586300 & 0.12836800\end{array}$




$\begin{array}{lrrr}\mathrm{H} & -2.03998100 & -0.86666900 & 0.61143700 \\ \mathrm{~N} & -1.64432300 & -1.53114800 & -1.27744900 \\ \mathrm{H} & -2.42668600 & -2.17220100 & -1.40282900 \\ \mathrm{H} & -2.67299700 & 1.04791200 & -0.79056300 \\ \mathrm{H} & -1.25788100 & -2.41813300 & 0.58030700 \\ \mathrm{H} & -0.83933400 & -1.92103400 & -1.76744900\end{array}$

I-TS (transition state between I and II)

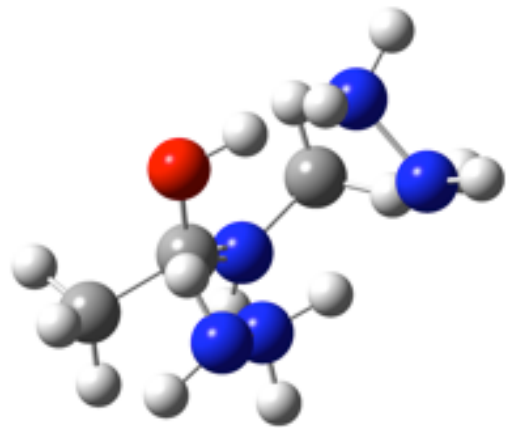

B3LYP/6-31G(d) free energy: -472.445287 (a.u.)

B3LYP/6-31G(d) thermal correction to Gibbs free energy: 0.190583 (a.u.)

Number of imaginary frequencies: 1 (-136.5844)

M06-2X/6-311+G(d,p)/SMD $\left(\mathrm{H}_{2} \mathrm{O}\right)$ single point energy: -472.673925977 (a.u.)

M06-2X/6-311+G(d,p)/SMD(EtOH) single point energy: -472.675588677 (a.u.)

M06-2X/6-311+G(d,p)/SMD $\left(\mathrm{CF}_{3} \mathrm{CH}_{2} \mathrm{OH}\right)$ single point energy: -472.674489159 (a.u.)

$\begin{array}{lrrc}\mathrm{C} & -1.09779500 & 0.27747700 & 0.21317000 \\ \mathrm{C} & -2.51484300 & -0.20931400 & 0.40483200 \\ \mathrm{H} & -2.52704900 & -1.24418400 & 0.75091100 \\ \mathrm{H} & -2.98866100 & 0.40745600 & 1.17454200 \\ \mathrm{H} & -3.09762000 & -0.12711700 & -0.51752800 \\ \mathrm{O} & -0.33928700 & 0.08919500 & 1.30805800 \\ \mathrm{H} & 0.63676700 & 0.28402600 & 1.16791200 \\ \mathrm{~N} & -0.97747500 & 1.45145400 & -0.47857900 \\ \mathrm{H} & -1.73928800 & 1.68477700 & -1.09936400 \\ \mathrm{C} & 0.12581200 & 2.39762700 & -0.40264100 \\ \mathrm{H} & 0.54112500 & 2.41302800 & 0.60777000 \\ \mathrm{H} & 0.92454600 & 2.18260000 & -1.12519000 \\ \mathrm{H} & -0.25839500 & 3.39696900 & -0.61817900 \\ \mathrm{H} & -0.17182200 & -2.30295300 & 0.65298000\end{array}$




$\begin{array}{cccc}\mathrm{H} & -1.26958700 & -2.81452000 & -0.43983200 \\ \mathrm{~N} & -0.36650800 & -1.08300800 & -0.88511100 \\ \mathrm{~N} & -0.33149400 & -2.42403000 & -0.34985700 \\ \mathrm{H} & -0.80195700 & -1.12043500 & -1.80985600 \\ \mathrm{~N} & 2.36850200 & -0.09035700 & 0.84848200 \\ \mathrm{H} & 2.37022900 & -1.03010900 & 1.24536000 \\ \mathrm{~N} & 2.48972600 & -0.24764600 & -0.58694200 \\ \mathrm{H} & 3.34748500 & -0.74839100 & -0.83229000 \\ \mathrm{H} & 0.62891300 & -0.81302400 & -1.02302000 \\ \mathrm{H} & 3.17972200 & 0.38926100 & 1.24901000 \\ \mathrm{H} & 2.58158900 & 0.68943400 & -0.97579700\end{array}$

II ( $\mathrm{MeC}(\mathrm{OH})\left(\mathrm{NH}_{2}{ }^{+} \mathrm{NH}_{2}\right)(\mathrm{NHMe})$ with $\left.\mathrm{H}_{2} \mathrm{NNH}_{2}\right)$

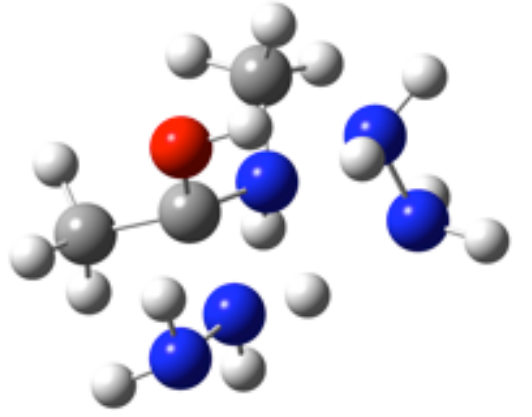

B3LYP/6-31G(d) free energy: -472.447151 (a.u.)

B3LYP/6-31G(d) thermal correction to Gibbs free energy: 0.194935 (a.u.)

Number of imaginary frequencies: 0

M06-2X/6-311+G(d,p)/SMD( $\left.\mathrm{H}_{2} \mathrm{O}\right)$ single point energy: -472.686950563 (a.u.)

M06-2X/6-311+G(d,p)/SMD(EtOH) single point energy: -472.688712619 (a.u.)

M06-2X/6-311+G(d,p)/SMD $\left(\mathrm{CF}_{3} \mathrm{CH}_{2} \mathrm{OH}\right)$ single point energy: -472.687736648 (a.u.)

$\begin{array}{lrrc}\mathrm{C} & -0.88703500 & 0.03943100 & 0.07101700 \\ \mathrm{C} & -2.38088700 & -0.20406900 & 0.23835000 \\ \mathrm{H} & -2.57772000 & -1.08191700 & 0.85941800 \\ \mathrm{H} & -2.81793500 & 0.65690400 & 0.75030000 \\ \mathrm{H} & -2.88065800 & -0.31994800 & -0.72973100 \\ \mathrm{O} & -0.27734800 & 0.03658900 & 1.29306200 \\ \mathrm{H} & 0.69100500 & 0.28547100 & 1.19577000 \\ \mathrm{~N} & -0.50049900 & 1.16934700 & -0.75492700 \\ \mathrm{H} & -1.07563800 & 1.18220900 & -1.59734500\end{array}$




$\begin{array}{lrrc}\mathrm{C} & -0.61650600 & 2.49157200 & -0.09833900 \\ \mathrm{H} & -1.62612600 & 2.71283700 & 0.27170500 \\ \mathrm{H} & 0.07910600 & 2.55239700 & 0.73949200 \\ \mathrm{H} & -0.34002300 & 3.25128900 & -0.83267200 \\ \mathrm{H} & -0.05955100 & -2.31205400 & 0.89933400 \\ \mathrm{H} & -1.29925100 & -2.78198500 & -0.06598600 \\ \mathrm{~N} & -0.25345800 & -1.20309900 & -0.71645600 \\ \mathrm{~N} & -0.32199400 & -2.48860600 & -0.07279300 \\ \mathrm{H} & -0.64909100 & -1.30438900 & -1.65656500 \\ \mathrm{~N} & 2.44546600 & 0.19889700 & 0.72895200 \\ \mathrm{H} & 2.78520400 & -0.64468500 & 1.18994100 \\ \mathrm{~N} & 2.36307400 & -0.08928500 & -0.68518300 \\ \mathrm{H} & 3.28232800 & -0.28041600 & -1.09057900 \\ \mathrm{H} & 0.77475300 & -0.96561200 & -0.83785000 \\ \mathrm{H} & 3.12702800 & 0.93623500 & 0.92439800 \\ \mathrm{H} & 1.98379800 & 0.74857300 & -1.12744200\end{array}$

II-TS (transition state between II and III)

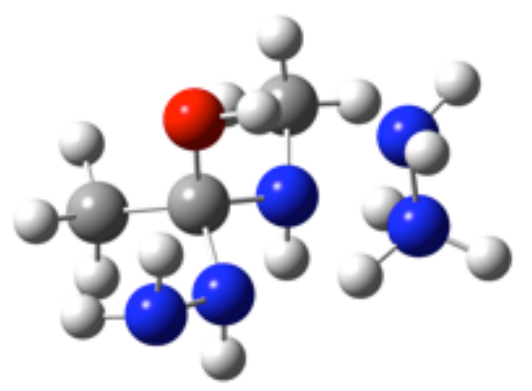

B3LYP/6-31G(d) free energy: -472.434056 (a.u.)

B3LYP/6-31G(d) thermal correction to Gibbs free energy: 0.194327 (a.u.)

Number of imaginary frequencies: 1 (-70.4908)

M06-2X/6-311+G(d,p)/SMD( $\left.\mathrm{H}_{2} \mathrm{O}\right)$ single point energy: -472.681514356 (a.u.)

M06-2X/6-311+G(d,p)/SMD(EtOH) single point energy: -472.683384792 (a.u.)

M06-2X/6-311+G(d,p)/SMD $\left(\mathrm{CF}_{3} \mathrm{CH}_{2} \mathrm{OH}\right)$ single point energy: -472.682405915 (a.u.)

$\begin{array}{lrrr}\mathrm{C} & -0.83670000 & 0.04951000 & 0.05603900 \\ \mathrm{C} & -2.35389500 & 0.21317400 & 0.08518600 \\ \mathrm{H} & -2.81831200 & -0.64705500 & 0.57441800 \\ \mathrm{H} & -2.63377500 & 1.09638200 & 0.66356900\end{array}$




$\begin{array}{lrrr}\mathrm{H} & -2.76025900 & 0.30667900 & -0.92771700 \\ \mathrm{O} & -0.39442200 & -0.02323200 & 1.36985300 \\ \mathrm{H} & 0.58193200 & -0.12910900 & 1.38067900 \\ \mathrm{~N} & -0.13307400 & 1.17541900 & -0.65691400 \\ \mathrm{H} & -0.50264900 & 1.23010500 & -1.60915900 \\ \mathrm{C} & -0.29861500 & 2.50859900 & -0.02507500 \\ \mathrm{H} & -1.32963600 & 2.87357400 & -0.07208500 \\ \mathrm{H} & 0.00244700 & 2.44255400 & 1.02100200 \\ \mathrm{H} & 0.34780100 & 3.22105000 & -0.54345100 \\ \mathrm{H} & -0.35858900 & -2.51485000 & 0.79615900 \\ \mathrm{H} & -1.79896000 & -2.45121900 & 0.02882200 \\ \mathrm{~N} & -0.37329900 & -1.18469500 & -0.67492600 \\ \mathrm{~N} & -0.78754400 & -2.44027700 & -0.12362800 \\ \mathrm{H} & -0.72905200 & -1.17427700 & -1.63232300 \\ \mathrm{~N} & 2.54796500 & -0.21511300 & 0.70133200 \\ \mathrm{H} & 3.12677700 & -1.03987700 & 0.85997000 \\ \mathrm{~N} & 2.17510800 & -0.16559400 & -0.68888100 \\ \mathrm{H} & 2.94850800 & -0.13068500 & -1.36430500 \\ \mathrm{H} & 1.49838500 & -0.96043100 & -0.85810400 \\ \mathrm{H} & 3.10631000 & 0.61086300 & 0.91489300 \\ \mathrm{H} & 1.40561500 & 0.63626500 & -0.78698100\end{array}$

III $\left(\mathrm{MeC}(\mathrm{OH})\left(\mathrm{NHNH}_{2}\right)\left(\mathrm{NH}_{2}^{+} \mathrm{Me}\right)\right.$ with $\left.\mathrm{H}_{2} \mathrm{NNH}_{2}\right)$

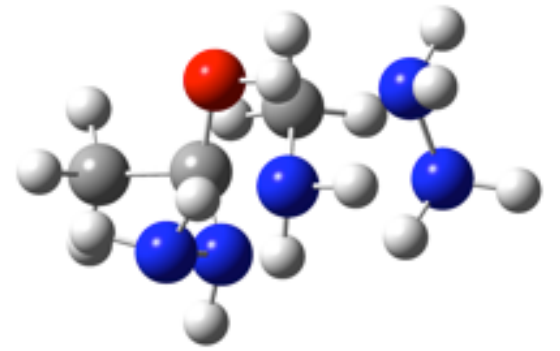

B3LYP/6-31G(d) free energy: -472.44771 (a.u.)

B3LYP/6-31G(d) thermal correction to Gibbs free energy: 0.195262 (a.u.)

Number of imaginary frequencies: 0

M06-2X/6-311+G(d,p)/SMD( $\left.\mathrm{H}_{2} \mathrm{O}\right)$ single point energy: -472.690810414 (a.u.)

M06-2X/6-311+G(d,p)/SMD(EtOH) single point energy: -472.692894749 (a.u.)

M06-2X/6-311+G(d,p)/SMD $\left(\mathrm{CF}_{3} \mathrm{CH}_{2} \mathrm{OH}\right)$ single point energy: -472.691796898 (a.u.) 


$\begin{array}{lrrr}\mathrm{C} & -0.86770500 & -0.08235600 & 0.08324600 \\ \mathrm{C} & -2.37483300 & 0.05620000 & 0.25108000 \\ \mathrm{H} & -2.76444600 & -0.86568100 & 0.69176800 \\ \mathrm{H} & -2.62718800 & 0.86901700 & 0.93602800 \\ \mathrm{H} & -2.87552900 & 0.21677400 & -0.70983000 \\ \mathrm{O} & -0.26569100 & -0.12068000 & 1.30374900 \\ \mathrm{H} & 0.71392800 & -0.31193500 & 1.19590800 \\ \mathrm{~N} & -0.30941500 & 1.18652100 & -0.68752800 \\ \mathrm{H} & -0.70101000 & 1.21588200 & -1.63427900 \\ \mathrm{C} & -0.53142000 & 2.49948100 & -0.00636600 \\ \mathrm{H} & -1.58141800 & 2.78228300 & -0.07787800 \\ \mathrm{H} & -0.24416000 & 2.38581700 & 1.03868900 \\ \mathrm{H} & 0.08906100 & 3.25443200 & -0.49222100 \\ \mathrm{H} & 0.18397700 & -2.63819300 & 0.44896900 \\ \mathrm{H} & -1.44563300 & -2.61805900 & 0.23902700 \\ \mathrm{~N} & -0.40810100 & -1.18491700 & -0.77791800 \\ \mathrm{~N} & -0.55005500 & -2.49703800 & -0.23891800 \\ \mathrm{H} & -0.91289700 & -1.18605100 & -1.66393100 \\ \mathrm{~N} & 2.46339200 & -0.17179600 & 0.73159800 \\ \mathrm{H} & 3.16518400 & -0.89751800 & 0.89667200 \\ \mathrm{~N} & 2.37243200 & 0.17176500 & -0.66954300 \\ \mathrm{H} & 3.29035500 & 0.37879100 & -1.07037300 \\ \mathrm{H} & 1.99576400 & -0.65315400 & -1.13928500 \\ \mathrm{H} & 2.77767100 & 0.66009000 & 1.22997000 \\ & 0.72784000 & 1.00124600 & -0.79082600\end{array}$

III-TS (transition state between III and IV)

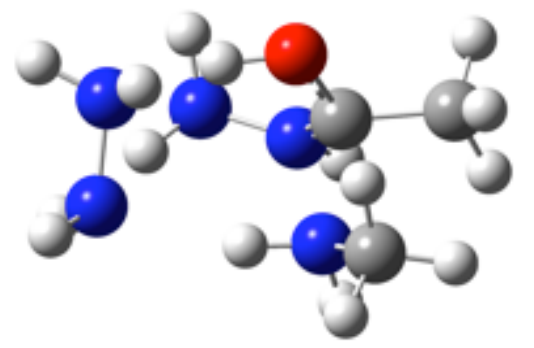

B3LYP/6-31G(d) free energy: -472.444928 (a.u.)

B3LYP/6-31G(d) thermal correction to Gibbs free energy: 0.190276 (a.u.) Number of imaginary frequencies: 1 (-198.6469) 
M06-2X/6-311+G(d,p)/SMD $\left(\mathrm{H}_{2} \mathrm{O}\right)$ single point energy: -472.670203103 (a.u.) M06-2X/6-311+G(d,p)/SMD(EtOH) single point energy: -472.673024407 (a.u.) M06-2X/6-311+G(d,p)/SMD( $\left.\mathrm{CF}_{3} \mathrm{CH}_{2} \mathrm{OH}\right)$ single point energy: -472.671633664 (a.u.)

$\begin{array}{lrrc}\mathrm{C} & -1.06879700 & -0.37403400 & 0.22775500 \\ \mathrm{C} & -2.48678500 & 0.03599600 & 0.53143900 \\ \mathrm{H} & -2.92603100 & -0.72153200 & 1.18904500 \\ \mathrm{H} & -2.50978800 & 0.99229000 & 1.05386600 \\ \mathrm{H} & -3.08984700 & 0.10100900 & -0.37870400 \\ \mathrm{O} & -0.24923700 & -0.29275300 & 1.27503300 \\ \mathrm{H} & 0.71004300 & -0.50488900 & 1.06038700 \\ \mathrm{~N} & -0.44176700 & 1.15580600 & -0.81852700 \\ \mathrm{H} & -0.85776400 & 1.09272000 & -1.74913100 \\ \mathrm{C} & -0.63665100 & 2.50108800 & -0.23652000 \\ \mathrm{H} & -1.69138700 & 2.77771900 & -0.28169200 \\ \mathrm{H} & -0.31918900 & 2.47489500 & 0.80892500 \\ \mathrm{H} & -0.05127800 & 3.25655700 & -0.77067000 \\ \mathrm{H} & 0.95614300 & -1.95917600 & -1.04221500 \\ \mathrm{H} & 0.30941200 & -2.69277900 & 0.31497100 \\ \mathrm{~N} & -0.93842800 & -1.43907900 & -0.62471200 \\ \mathrm{~N} & 0.11741200 & -2.35044500 & -0.62407800 \\ \mathrm{H} & -1.75239500 & -1.74591700 & -1.14082900 \\ \mathrm{~N} & 2.43462700 & -0.04921300 & 0.83112000 \\ \mathrm{H} & 3.28046700 & -0.54346600 & 1.12981900 \\ \mathrm{~N} & 2.55273100 & 0.45569700 & -0.52220600 \\ \mathrm{H} & 3.38554600 & 1.04207800 & -0.62265000 \\ \mathrm{H} & 2.70889100 & -0.35213500 & -1.12383800 \\ \mathrm{H} & 2.35161000 & 0.76566900 & 1.43923800 \\ \mathrm{H} & 0.57083400 & 0.97132000 & -0.91401600\end{array}$

IV $\left(\mathrm{AcNHNH}_{2}\right.$ with $\mathrm{MeNH}_{2}$ and $\left.\mathrm{H}_{2} \mathrm{NNH}_{3}{ }^{+}\right)$ 


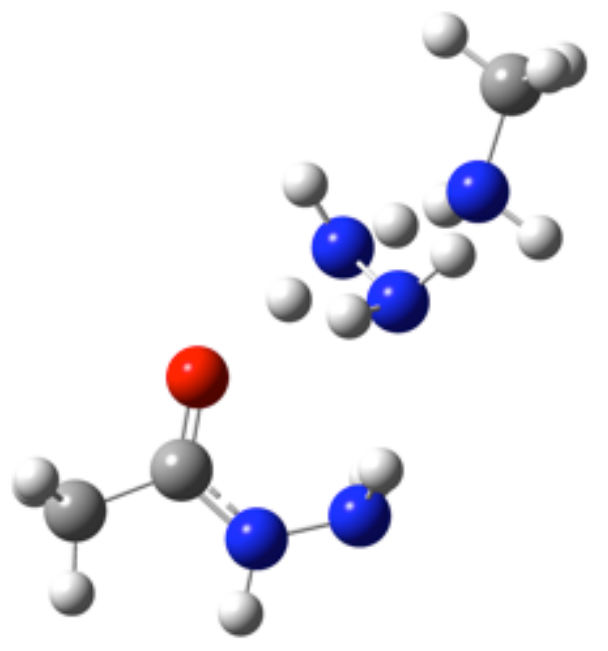

B3LYP/6-31G(d) free energy: -472.493359 (a.u.)

B3LYP/6-31G(d) thermal correction to Gibbs free energy: 0.18294 (a.u.)

Number of imaginary frequencies: 0

M06-2X/6-311+G(d,p)/SMD $\left(\mathrm{H}_{2} \mathrm{O}\right)$ single point energy: -472.699827063 (a.u.)

M06-2X/6-311+G(d,p)/SMD(EtOH) single point energy: -472.702869937 (a.u.)

M06-2X/6-311+G(d,p)/SMD $\left(\mathrm{CF}_{3} \mathrm{CH}_{2} \mathrm{OH}\right)$ single point energy: -472.701076038 (a.u.)

$\begin{array}{lrrr}\mathrm{C} & -2.36570100 & 0.30444500 & -0.30265100 \\ \mathrm{C} & -3.68990200 & 0.99622300 & -0.51458400 \\ \mathrm{H} & -3.82082300 & 1.76081300 & 0.25678600 \\ \mathrm{H} & -3.66822200 & 1.50816600 & -1.48172600 \\ \mathrm{H} & -4.54360900 & 0.31407500 & -0.49217500 \\ \mathrm{O} & -1.30415600 & 0.95363400 & -0.26882600 \\ \mathrm{H} & 0.21755900 & 0.77071500 & 0.24960900 \\ \mathrm{~N} & 3.10900800 & -0.34283000 & -0.85448800 \\ \mathrm{H} & 2.81259500 & -0.26122200 & -1.82852900 \\ \mathrm{C} & 4.45228100 & 0.27246400 & -0.70345000 \\ \mathrm{H} & 4.38535300 & 1.34013100 & -0.92774900 \\ \mathrm{H} & 4.78803000 & 0.15521800 & 0.33029400 \\ \mathrm{H} & 5.20120300 & -0.17624800 & -1.36475000 \\ \mathrm{H} & -0.68706500 & -1.76049600 & -0.83577300 \\ \mathrm{H} & -0.74007600 & -1.57006400 & 0.80490500 \\ \mathrm{~N} & -2.40348000 & -1.03723200 & -0.15930800 \\ \mathrm{~N} & -1.27803100 & -1.86171200 & -0.01156600 \\ \mathrm{H} & -3.28434700 & -1.53260800 & -0.19878900\end{array}$




$\begin{array}{cccc}\mathrm{N} & 1.14877500 & 0.74773100 & 0.76321100 \\ \mathrm{H} & 1.40947700 & 1.71072500 & 1.01417000 \\ \mathrm{~N} & 0.95779800 & -0.13346300 & 1.89166300 \\ \mathrm{H} & 1.84741000 & -0.22249500 & 2.38171100 \\ \mathrm{H} & 0.29922200 & 0.31515000 & 2.52818100 \\ \mathrm{H} & 1.92137100 & 0.31938000 & 0.11025400 \\ \mathrm{H} & 3.17661500 & -1.34656500 & -0.67828800\end{array}$

Structures for the Reaction Pathway in the Absence of Hydrazinium Salt I' (AcNHMe with $2 \mathrm{H}_{2} \mathrm{NNH}_{2}$ )

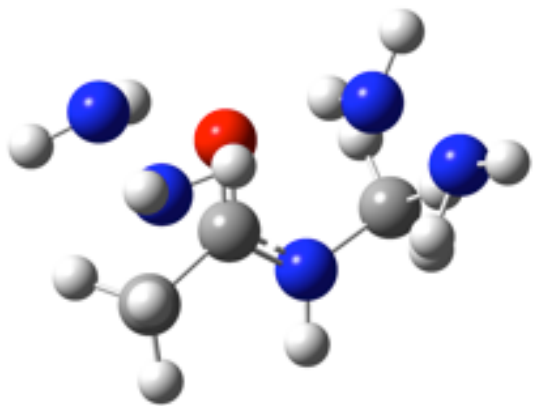

B3LYP/6-31G(d) free energy: -472.084952 (a.u.)

B3LYP/6-31G(d) thermal correction to Gibbs free energy: 0.170708 (a.u.)

Number of imaginary frequencies: 0

M06-2X/6-311+G(d,p)/SMD $\left(\mathrm{H}_{2} \mathrm{O}\right)$ single point energy: -472.2235715 (a.u.)

$\begin{array}{lrrr}\mathrm{C} & 0.77037400 & 1.16912700 & 0.08153600 \\ \mathrm{C} & -0.00427700 & 2.15872700 & -0.76476900 \\ \mathrm{H} & -0.87802100 & 1.63571000 & -1.17775200 \\ \mathrm{H} & -0.37313600 & 2.96297700 & -0.12043300 \\ \mathrm{H} & 0.58662800 & 2.59783500 & -1.57522600 \\ \mathrm{O} & 0.26631100 & 0.61909200 & 1.06930600 \\ \mathrm{H} & -0.21119600 & -1.37163400 & 0.73684600 \\ \mathrm{~N} & 2.04092800 & 0.89586300 & -0.30761800 \\ \mathrm{H} & 2.40566000 & 1.35169900 & -1.13040300 \\ \mathrm{C} & 2.87404200 & -0.07826300 & 0.38893700 \\ \mathrm{H} & 2.80572600 & 0.08909000 & 1.46699600 \\ \mathrm{H} & 2.55071600 & -1.10411500 & 0.17376800 \\ \mathrm{H} & 3.91043800 & 0.05445300 & 0.06918100 \\ \mathrm{H} & -1.96121400 & 0.63028700 & 1.11465200\end{array}$




$\begin{array}{lrrr}\mathrm{H} & -3.16450900 & 1.53123100 & 0.56430700 \\ \mathrm{~N} & -2.64539500 & 0.04083900 & -0.65356000 \\ \mathrm{~N} & -2.90340300 & 0.55372000 & 0.71897700 \\ \mathrm{H} & -3.56615500 & -0.27719300 & -0.96639100 \\ \mathrm{~N} & 0.60515300 & -2.61796300 & -0.58284600 \\ \mathrm{H} & 0.31367200 & -3.50941200 & -0.99147300 \\ \mathrm{~N} & -0.55516700 & -2.20268200 & 0.24430700 \\ \mathrm{H} & -2.13713600 & -0.83629000 & -0.48428000 \\ \mathrm{H} & -0.60699500 & -2.91236300 & 0.97830800 \\ \mathrm{H} & 0.55938400 & -1.98099000 & -1.38159300\end{array}$

$\mathbf{I}^{\prime}$-TS (transition state between $\mathbf{I}^{\prime}$ and $\mathbf{I I}^{\prime}$ )

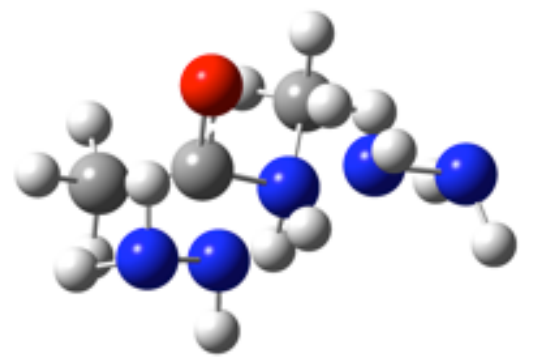

B3LYP/6-31G(d) free energy: -472.02777 (a.u.)

B3LYP/6-31G(d) thermal correction to Gibbs free energy: 0.176895 (a.u.) Number of imaginary frequencies: 1 (-904.4107)

M06-2X/6-311+G(d,p)/SMD $\left(\mathrm{H}_{2} \mathrm{O}\right)$ single point energy: -472.192590351 (a.u.)

$\begin{array}{lrrr}\mathrm{C} & -0.79199900 & 0.19846300 & 0.10479200 \\ \mathrm{C} & -2.30680600 & 0.46414000 & -0.06237600 \\ \mathrm{H} & -2.88984300 & -0.32219400 & 0.42778400 \\ \mathrm{H} & -2.55855800 & 1.40274800 & 0.43998300 \\ \mathrm{H} & -2.62124000 & 0.53682600 & -1.11337200 \\ \mathrm{O} & -0.40477000 & 0.02186400 & 1.34188700 \\ \mathrm{H} & 1.27711900 & -0.43126300 & 1.07757200 \\ \mathrm{~N} & 0.03114400 & 1.22476600 & -0.63755000 \\ \mathrm{H} & -0.38231200 & 1.39170200 & -1.55726000 \\ \mathrm{C} & 0.09583400 & 2.50574800 & 0.08081600 \\ \mathrm{H} & -0.86657000 & 3.03980300 & 0.13852500 \\ \mathrm{H} & 0.42779700 & 2.30046600 & 1.09990400 \\ \mathrm{H} & 0.82099500 & 3.16447000 & -0.41012500\end{array}$




$\begin{array}{lrrr}\mathrm{H} & -0.88369900 & -2.17214700 & 0.85957800 \\ \mathrm{H} & -2.05369800 & -2.21187000 & -0.25256600 \\ \mathrm{~N} & -0.41800600 & -1.13414100 & -0.70191000 \\ \mathrm{~N} & -1.03932800 & -2.31346800 & -0.14546500 \\ \mathrm{H} & -0.62724300 & -1.10970200 & -1.70159400 \\ \mathrm{~N} & 2.82568600 & 0.00037900 & -0.22090300 \\ \mathrm{H} & 3.15765300 & -0.45629300 & -1.07413600 \\ \mathrm{~N} & 1.88109500 & -0.94112900 & 0.37017300 \\ \mathrm{H} & 0.90622700 & -1.21758200 & -0.33679100 \\ \mathrm{H} & 2.39959300 & -1.69000800 & 0.82925200 \\ \mathrm{H} & 2.18562700 & 0.73518900 & -0.55165400\end{array}$

II' $\left(\mathrm{MeC}(\mathrm{OH})\left(\mathrm{NHNH}_{2}\right)(\mathrm{NHMe})\right.$ with $\left.\mathrm{H}_{2} \mathrm{NNH}_{2}\right)$

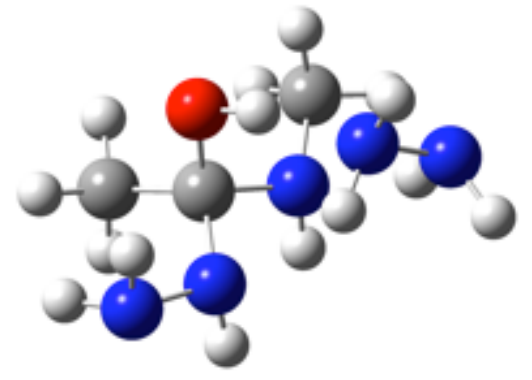

B3LYP/6-31G(d) free energy: -472.059669 (a.u.)

B3LYP/6-31G(d) thermal correction to Gibbs free energy: 0.178676 (a.u.)

Number of imaginary frequencies: 0

M06-2X/6-311+G(d,p)/SMD( $\left.\mathrm{H}_{2} \mathrm{O}\right)$ single point energy: -472.224655047 (a.u.)

$\begin{array}{lrrr}\mathrm{C} & -0.82378000 & 0.09190000 & 0.04337000 \\ \mathrm{C} & -2.32085400 & 0.35546200 & 0.28200300 \\ \mathrm{H} & -2.79164300 & -0.48562500 & 0.80023300 \\ \mathrm{H} & -2.45696700 & 1.23430400 & 0.91742300 \\ \mathrm{H} & -2.84459100 & 0.52322800 & -0.66704400 \\ \mathrm{O} & -0.24227100 & -0.09762500 & 1.29279300 \\ \mathrm{H} & 0.72482400 & -0.30600100 & 1.13684800 \\ \mathrm{~N} & -0.15043300 & 1.19869900 & -0.67415800 \\ \mathrm{H} & -0.55998800 & 1.29533400 & -1.60323300 \\ \mathrm{C} & -0.17871400 & 2.49674300 & 0.00726100 \\ \mathrm{H} & -1.17733200 & 2.95634400 & 0.08235500\end{array}$




$\begin{array}{lrrr}\mathrm{H} & 0.21379900 & 2.36331000 & 1.01718500 \\ \mathrm{H} & 0.47726700 & 3.18981100 & -0.52900200 \\ \mathrm{H} & -0.60883500 & -2.56936000 & 0.51774900 \\ \mathrm{H} & -2.08963900 & -2.20817300 & -0.04511800 \\ \mathrm{~N} & -0.54768600 & -1.10641600 & -0.79298100 \\ \mathrm{~N} & -1.11438800 & -2.33730500 & -0.33517100 \\ \mathrm{H} & -0.87951500 & -0.96798800 & -1.74605000 \\ \mathrm{~N} & 2.79360100 & 0.23332200 & -0.37462400 \\ \mathrm{H} & 3.25391700 & -0.18449100 & -1.18605300 \\ \mathrm{~N} & 2.36775700 & -0.80346500 & 0.53237600 \\ \mathrm{H} & 2.10586000 & -1.65872100 & 0.03762400 \\ \mathrm{H} & 3.14831900 & -1.01555600 & 1.14989100 \\ \mathrm{H} & 1.92081900 & 0.65610000 & -0.70904500\end{array}$

II'-TS (transition state between $\mathbf{I I}^{\prime}$ and $\mathbf{I I}^{\prime \prime}$ )

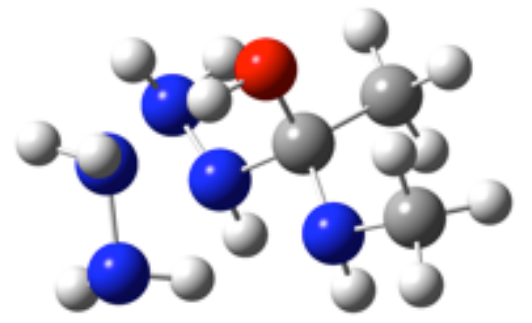

B3LYP/6-31G(d) free energy: -472.056994 (a.u.)

B3LYP/6-31G(d) thermal correction to Gibbs free energy: 0.178586 (a.u.) Number of imaginary frequencies: 1 (-185.1234)

M06-2X/6-311+G(d,p)/SMD( $\left.\mathrm{H}_{2} \mathrm{O}\right)$ single point energy: -472.220741456 (a.u.)

$\begin{array}{lrrr}\mathrm{C} & -0.83111300 & 0.01597200 & 0.04753900 \\ \mathrm{C} & -2.34484700 & 0.12240400 & 0.30409200 \\ \mathrm{H} & -2.72031900 & -0.77048600 & 0.81415800 \\ \mathrm{H} & -2.56662400 & 0.97389600 & 0.95261600 \\ \mathrm{H} & -2.89278500 & 0.24553000 & -0.63801500 \\ \mathrm{O} & -0.21831800 & -0.10477500 & 1.29012300 \\ \mathrm{H} & 0.77093400 & -0.15639600 & 1.13560600 \\ \mathrm{~N} & -0.27986500 & 1.17973200 & -0.68143600 \\ \mathrm{H} & -0.65735800 & 1.19563400 & -1.62862600 \\ \mathrm{C} & -0.49583300 & 2.48193100 & -0.04528400\end{array}$




$\begin{array}{lrcc}\mathrm{H} & -1.54374200 & 2.82245100 & -0.03166300 \\ \mathrm{H} & -0.14364000 & 2.42171600 & 0.98693500 \\ \mathrm{H} & 0.10076800 & 3.23476600 & -0.57054400 \\ \mathrm{H} & -0.19643300 & -2.58111900 & 0.53169500 \\ \mathrm{H} & -1.75705800 & -2.42671400 & 0.11097900 \\ \mathrm{~N} & -0.45067900 & -1.15194900 & -0.79123300 \\ \mathrm{~N} & -0.80499800 & -2.43585700 & -0.27128100 \\ \mathrm{H} & -0.87929100 & -1.08542800 & -1.71333500 \\ \mathrm{~N} & 2.69287000 & 0.16153000 & -0.68404400 \\ \mathrm{H} & 2.61048900 & -0.68623600 & -1.24668500 \\ \mathrm{~N} & 2.52651100 & -0.28436400 & 0.71247000 \\ \mathrm{H} & 2.85421700 & -1.24822800 & 0.77205000 \\ \mathrm{H} & 3.20929500 & 0.25323600 & 1.24738900 \\ \mathrm{H} & 1.80197400 & 0.64008800 & -0.87295800\end{array}$

II' $\left(\mathrm{MeC}(\mathrm{OH})\left(\mathrm{NHNH}_{2}\right)(\mathrm{NHMe})\right.$ with partially-rotated $\left.\mathrm{H}_{2} \mathrm{NNH}_{2}\right)$

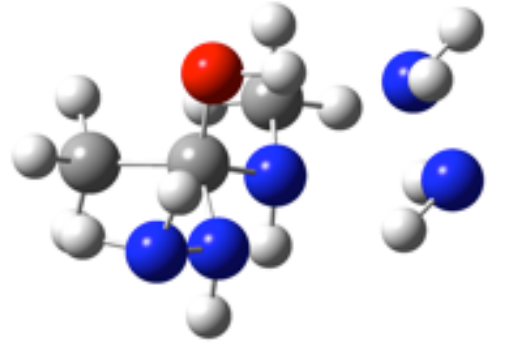

B3LYP/6-31G(d) free energy: -472.057987 (a.u.)

B3LYP/6-31G(d) thermal correction to Gibbs free energy: 0.178271 (a.u.) Number of imaginary frequencies: 0

M06-2X/6-311+G(d,p)/SMD( $\left.\mathrm{H}_{2} \mathrm{O}\right)$ single point energy: -472.218879974 (a.u.)

$\begin{array}{lrrc}\mathrm{C} & -0.81593600 & -0.01615000 & 0.05225800 \\ \mathrm{C} & -2.33511500 & -0.00536600 & 0.29987600 \\ \mathrm{H} & -2.61563100 & 0.84233100 & 0.93018600 \\ \mathrm{H} & -2.65416500 & -0.91017100 & 0.82688100 \\ \mathrm{H} & -2.88503000 & 0.06303100 & -0.64663100 \\ \mathrm{O} & -0.20423000 & -0.07416800 & 1.29904000 \\ \mathrm{H} & 0.78830100 & -0.06542700 & 1.15257800 \\ \mathrm{~N} & -0.36107900 & -1.17855000 & -0.76214000 \\ \mathrm{H} & -0.79207600 & -1.15833300 & -1.68550300\end{array}$




$\begin{array}{lrrr}\mathrm{H} & -1.58824300 & -2.51034700 & 0.16706700 \\ \mathrm{H} & -0.02084300 & -2.56279900 & 0.59009200 \\ \mathrm{H} & -0.31692800 & 2.45160400 & 0.94333000 \\ \mathrm{H} & -1.73021600 & 2.72517300 & -0.09767600 \\ \mathrm{~N} & -0.33866900 & 1.16688000 & -0.69823300 \\ \mathrm{H} & -0.70728400 & 1.13456500 & -1.64855700 \\ \mathrm{~N} & 2.63760000 & 0.04342400 & -0.75093900 \\ \mathrm{H} & 1.91509200 & 0.74063800 & -0.97093900 \\ \mathrm{~N} & 2.54070100 & -0.02478600 & 0.72835800 \\ \mathrm{H} & 3.04636600 & 0.80069400 & 1.05487700 \\ \mathrm{H} & 3.14912200 & -0.80079200 & 0.99534600 \\ \mathrm{H} & 2.13792900 & -0.80412500 & -1.03932900 \\ \mathrm{~N} & -0.63718300 & -2.47062400 & -0.21469400 \\ \mathrm{C} & -0.65993600 & 2.46242200 & -0.09390900 \\ \mathrm{H} & -0.11622300 & 3.24745900 & -0.62984700\end{array}$

II'-TS (transition state between II" and III')

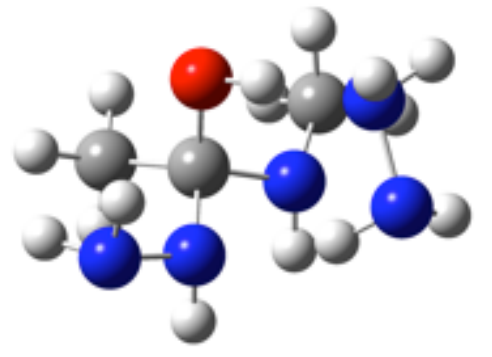

B3LYP/6-31G(d) free energy: -472.056783 (a.u.)

B3LYP/6-31G(d) thermal correction to Gibbs free energy: 0.17846 (a.u.)

Number of imaginary frequencies: 1 (-196.7866)

M06-2X/6-311+G(d,p)/SMD( $\left.\mathrm{H}_{2} \mathrm{O}\right)$ single point energy: -472.220973244 (a.u.)

$\begin{array}{lrrc}\mathrm{C} & 0.82602200 & 0.07064300 & 0.04913400 \\ \mathrm{C} & 2.33526800 & 0.24472600 & 0.29656600 \\ \mathrm{H} & 2.72180600 & -0.58483000 & 0.89422800 \\ \mathrm{H} & 2.54228400 & 1.16171100 & 0.85754300 \\ \mathrm{H} & 2.88502900 & 0.27976800 & -0.65184300 \\ \mathrm{O} & 0.21469600 & 0.02517400 & 1.29692400 \\ \mathrm{H} & -0.76818500 & -0.10210200 & 1.14526200 \\ \mathrm{~N} & 0.22887000 & 1.19032200 & -0.73658900\end{array}$




$\begin{array}{lrrr}\mathrm{H} & 0.64711500 & 1.23801200 & -1.66488800 \\ \mathrm{H} & 1.28780200 & 2.63362300 & 0.22735400 \\ \mathrm{H} & -0.28300400 & 2.50923300 & 0.62824100 \\ \mathrm{H} & 0.59137600 & -2.45431700 & 0.88103300 \\ \mathrm{H} & 2.03864900 & -2.54838100 & -0.14308500 \\ \mathrm{~N} & 0.49103400 & -1.13628600 & -0.73001600 \\ \mathrm{H} & 0.86111700 & -1.04261200 & -1.67534200 \\ \mathrm{~N} & -2.70338900 & 0.03422800 & -0.69667100 \\ \mathrm{H} & -2.53642000 & -0.83049200 & -1.21273300 \\ \mathrm{~N} & -2.51007900 & -0.32314800 & 0.72183200 \\ \mathrm{H} & -2.75349100 & -1.30747000 & 0.82993600 \\ \mathrm{H} & -3.24142200 & 0.17996800 & 1.22529600 \\ \mathrm{H} & -1.85838400 & 0.58027500 & -0.90984500 \\ \mathrm{~N} & 0.35187300 & 2.49611100 & -0.16711900 \\ \mathrm{C} & 0.94560100 & -2.40099100 & -0.15131500 \\ \mathrm{H} & 0.49864000 & -3.22862800 & -0.71291400\end{array}$

III' $\left(\mathrm{MeC}(\mathrm{OH})\left(\mathrm{NHNH}_{2}\right)(\mathrm{NHMe})\right.$ with rotated $\left.\mathrm{H}_{2} \mathrm{NNH}_{2}\right)$

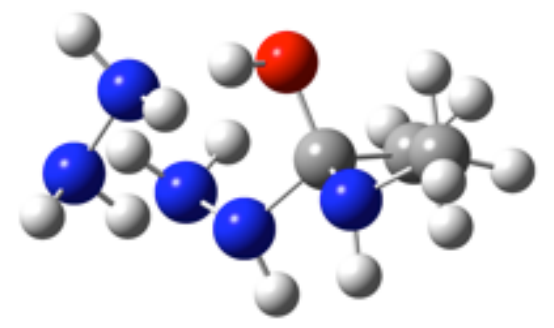

B3LYP/6-31G(d) free energy: -472.060077 (a.u.)

B3LYP/6-31G(d) thermal correction to Gibbs free energy: 0.178754 (a.u.)

Number of imaginary frequencies: 0

M06-2X/6-311+G(d,p)/SMD( $\left.\mathrm{H}_{2} \mathrm{O}\right)$ single point energy: -472.225108634 (a.u.)

$\begin{array}{lrrc}\mathrm{C} & 0.79958900 & 0.26210100 & 0.05238500 \\ \mathrm{C} & 2.16749600 & 0.91176900 & 0.32949600 \\ \mathrm{H} & 2.77457700 & 0.27848000 & 0.98190400 \\ \mathrm{H} & 2.04452800 & 1.87514700 & 0.83619100 \\ \mathrm{H} & 2.71491300 & 1.07921600 & -0.60594100 \\ \mathrm{O} & 0.20663500 & 0.03372300 & 1.28746300 \\ \mathrm{H} & -0.66283100 & -0.42895100 & 1.11633200\end{array}$




$\begin{array}{lrrr}\mathrm{N} & -0.07283200 & 1.15424200 & -0.77638300 \\ \mathrm{H} & 0.46499100 & 1.50564200 & -1.56820200 \\ \mathrm{H} & 0.02294900 & 2.58415900 & 0.65575800 \\ \mathrm{H} & -1.46176900 & 1.99818400 & 0.33780600 \\ \mathrm{H} & 1.61893100 & -2.16750300 & 0.83050500 \\ \mathrm{H} & 2.88136800 & -1.71276200 & -0.33489700 \\ \mathrm{~N} & 0.86374500 & -0.99229800 & -0.71529400 \\ \mathrm{H} & 1.03718800 & -0.78376800 & -1.69709900 \\ \mathrm{~N} & -2.73394400 & -0.25210000 & -0.43213900 \\ \mathrm{H} & -3.27715000 & -0.75250800 & -1.13876800 \\ \mathrm{~N} & -2.22505100 & -1.16820700 & 0.55484100 \\ \mathrm{H} & -1.93972500 & -2.06233100 & 0.14967600 \\ \mathrm{H} & -2.96737800 & -1.34523800 & 1.22773400 \\ \mathrm{H} & -1.90422100 & 0.13501500 & -0.89623700 \\ \mathrm{~N} & -0.59899300 & 2.30636200 & -0.10788200 \\ \mathrm{C} & 1.81825200 & -1.98749700 & -0.22958000 \\ \mathrm{H} & 1.65805400 & -2.92679700 & -0.77026800\end{array}$

III'-TS (transition state between III' and IV')

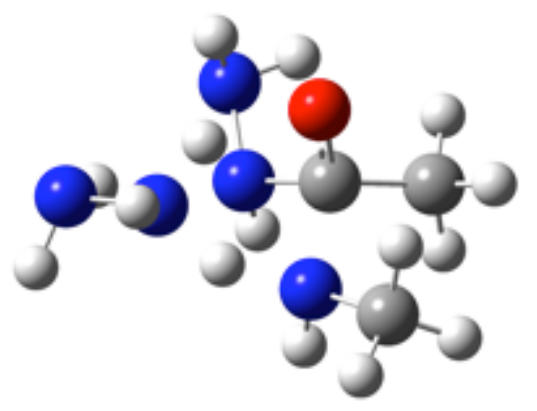

B3LYP/6-31G(d) free energy: -472.028124 (a.u.)

B3LYP/6-31G(d) thermal correction to Gibbs free energy: 0.176753 (a.u.)

Number of imaginary frequencies: 1 (-972.4533)

M06-2X/6-311+G(d,p)/SMD $\left(\mathrm{H}_{2} \mathrm{O}\right)$ single point energy: -472.194268233 (a.u.)

$\begin{array}{lrrr}\mathrm{C} & 0.76414600 & 0.25675600 & 0.12540900 \\ \mathrm{C} & 2.26678800 & 0.58938100 & -0.01379900 \\ \mathrm{H} & 2.86471700 & -0.10135200 & 0.58698300 \\ \mathrm{H} & 2.44934700 & 1.59364700 & 0.38454600 \\ \mathrm{H} & 2.62311000 & 0.55847100 & -1.05299300\end{array}$




$\begin{array}{lrrr}\mathrm{O} & 0.33688600 & 0.16821000 & 1.35514000 \\ \mathrm{H} & -1.26571700 & -0.41103700 & 1.08744300 \\ \mathrm{~N} & -0.09638500 & 1.20908400 & -0.69233700 \\ \mathrm{H} & 0.32130800 & 1.39804700 & -1.60533200 \\ \mathrm{H} & 0.68759300 & 2.85155300 & 0.19899900 \\ \mathrm{H} & -0.66774000 & 2.26953800 & 0.84041700 \\ \mathrm{H} & 1.19281000 & -2.18798200 & 0.96864200 \\ \mathrm{H} & 2.30371000 & -2.22037000 & -0.42297200 \\ \mathrm{~N} & 0.47434300 & -1.11662200 & -0.65190800 \\ \mathrm{H} & 0.62240400 & -1.01679900 & -1.65796500 \\ \mathrm{~N} & -2.83170900 & -0.11252200 & -0.23053600 \\ \mathrm{H} & -3.12637000 & -0.60956800 & -1.07461000 \\ \mathrm{~N} & -1.84591500 & -0.98247200 & 0.40248600 \\ \mathrm{H} & -0.83759600 & -1.24608600 & -0.27793100 \\ \mathrm{H} & -2.33461600 & -1.72650500 & 0.90064600 \\ \mathrm{H} & -2.23597400 & 0.65173300 & -0.57667400 \\ \mathrm{~N} & -0.24240200 & 2.49660400 & -0.05964400 \\ \mathrm{C} & 1.25006000 & -2.24786100 & -0.12169900 \\ \mathrm{H} & 0.81643600 & -3.19712900 & -0.45621600\end{array}$

IV' $\left(\mathrm{AcNHNH}_{2}\right.$ with $\mathrm{MeNH}_{2}$ and $\left.\mathrm{H}_{2} \mathrm{NNH}_{2}\right)$

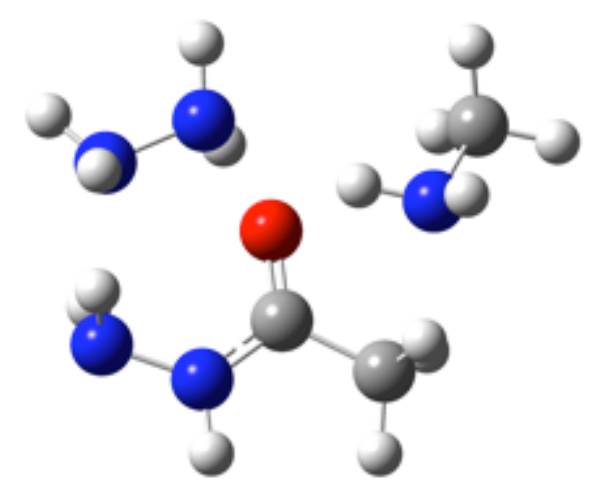

B3LYP/6-31G(d) free energy: -472.094091 (a.u.)

B3LYP/6-31G(d) thermal correction to Gibbs free energy: 0.1727 (a.u.)

Number of imaginary frequencies: 0

M06-2X/6-311+G(d,p)/SMD( $\left.\mathrm{H}_{2} \mathrm{O}\right)$ single point energy: -472.232965636 (a.u.)
C
$-0.61639100$
1.32984500
0.26049700
$\mathrm{C}$
0.30027900
2.42713200
$-0.23502900$ 


$\begin{array}{lrrc}\mathrm{H} & 1.15754900 & 1.93823500 & -0.71746000 \\ \mathrm{H} & 0.66619100 & 3.00080000 & 0.62079200 \\ \mathrm{H} & -0.18133500 & 3.10631700 & -0.94654800 \\ \mathrm{O} & -0.33277300 & 0.61347200 & 1.22768700 \\ \mathrm{H} & -0.01735400 & -1.28722900 & 0.75140900 \\ \mathrm{~N} & -1.76232700 & 1.15505500 & -0.44748100 \\ \mathrm{H} & -2.00907900 & 1.78221700 & -1.19876100 \\ \mathrm{H} & -2.89375200 & 0.21046900 & 0.84729100 \\ \mathrm{H} & -2.27828900 & -0.75417600 & -0.32541900 \\ \mathrm{H} & 2.40273700 & 0.26222300 & 1.13843200 \\ \mathrm{H} & 3.92922800 & 0.68936600 & 0.35133800 \\ \mathrm{~N} & 2.46525400 & -0.15325800 & -0.90726000 \\ \mathrm{H} & 3.12061400 & -0.49686300 & -1.60810200 \\ \mathrm{~N} & -1.16092500 & -2.53587100 & -0.29137100 \\ \mathrm{H} & -1.64045100 & -3.16588100 & 0.35518100 \\ \mathrm{~N} & 0.13032500 & -2.13972100 & 0.20489800 \\ \mathrm{H} & 1.71778700 & -0.84884900 & -0.84175500 \\ \mathrm{H} & 0.52249700 & -2.84985100 & 0.82532900 \\ \mathrm{H} & -1.01209100 & -3.05454000 & -1.15415700 \\ \mathrm{~N} & -2.71919100 & 0.16227600 & -0.15768600 \\ \mathrm{C} & 3.13544900 & -0.06584600 & 0.39421100 \\ \mathrm{H} & 3.58995300 & -1.00616700 & 0.75516500\end{array}$




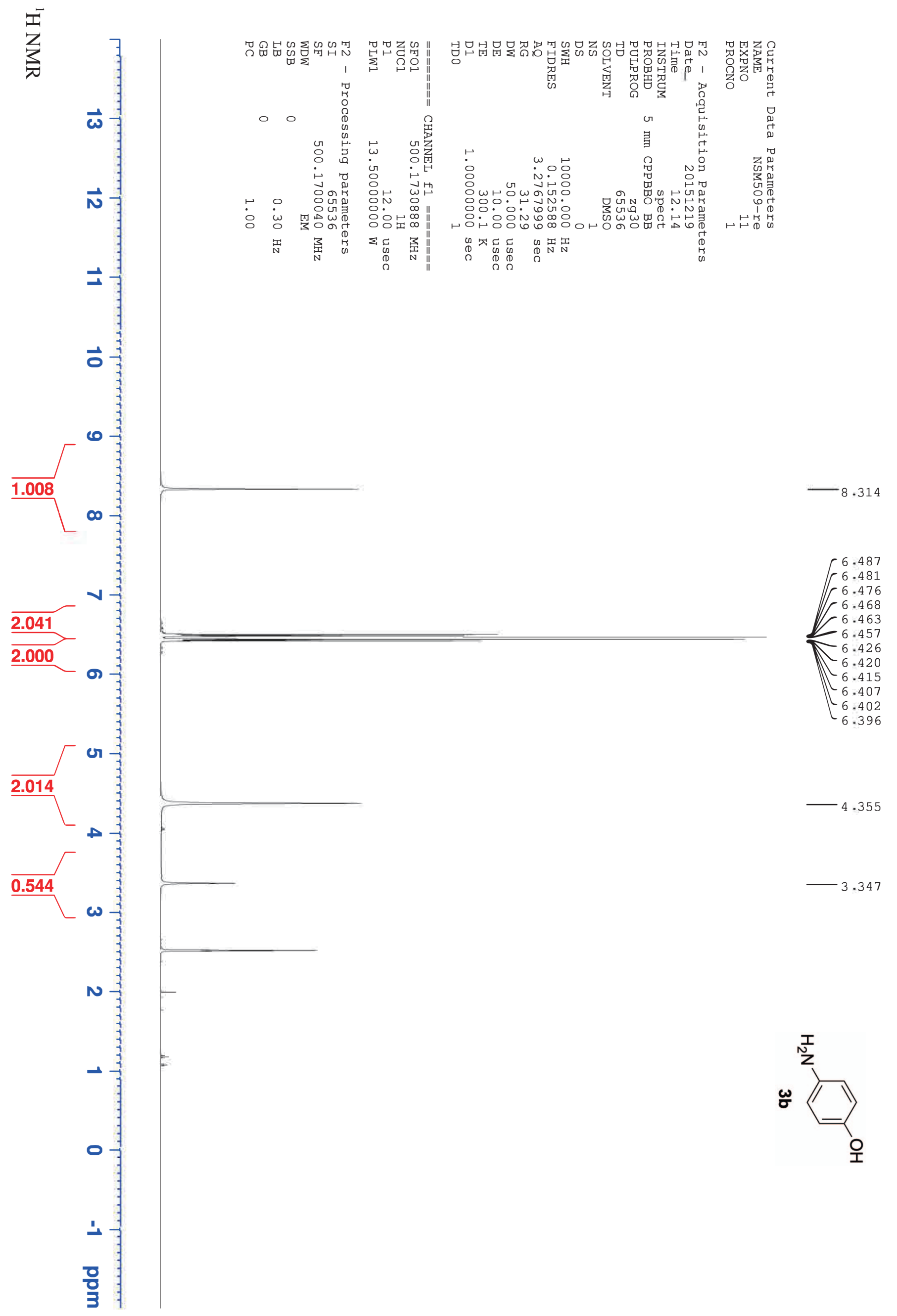




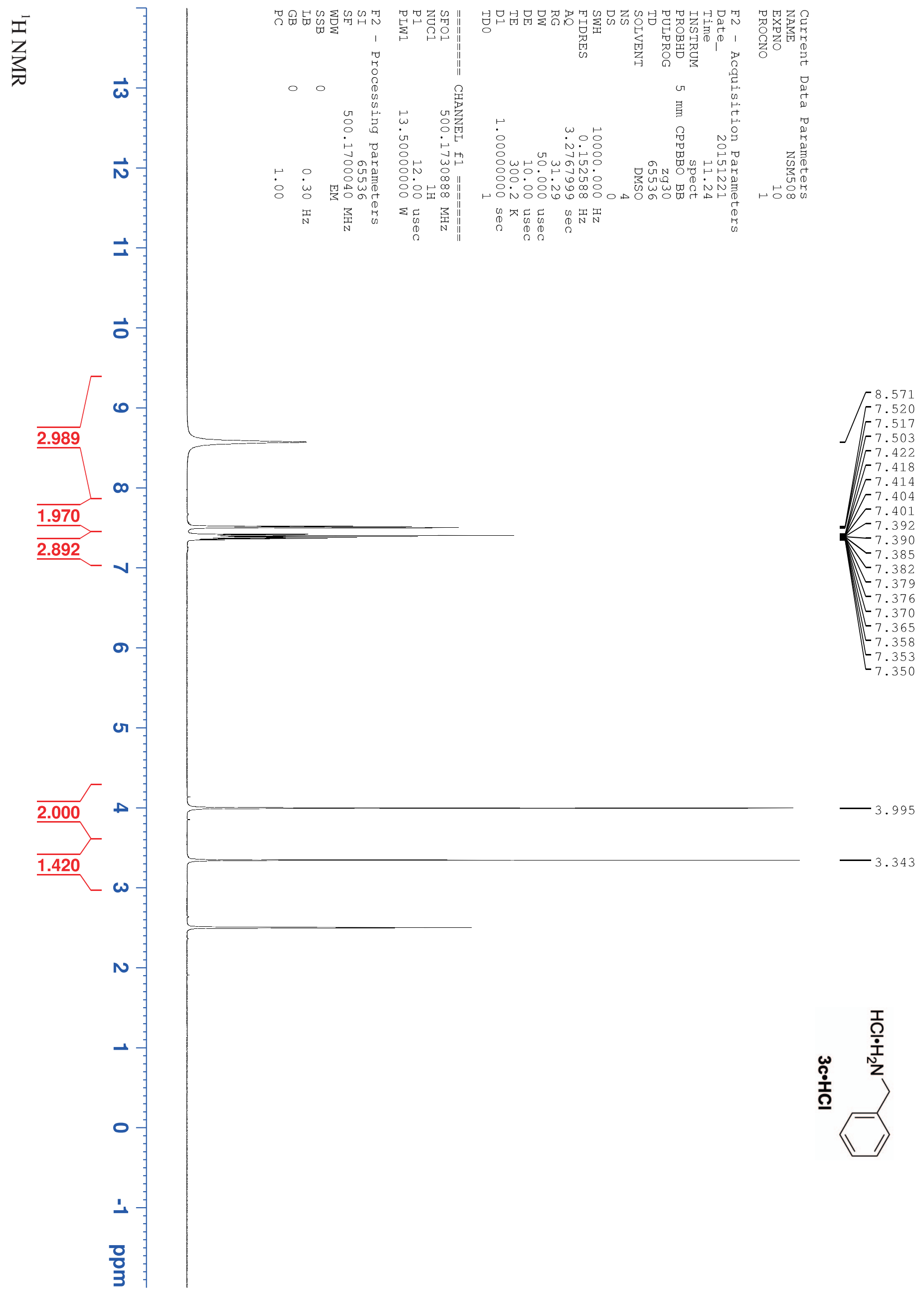

